INGRAM, G. I. C. (1951). J. gen. Microbiol. 5, 30-38.

\title{
Classification of Staphylococci by Penicillin Lysis
}

\author{
BY G. I. C. INGRAM \\ Department of Pathology, St Thomas's Hospital, Hydestile, Surrey
}

SUMMARY : In twenty-nine of a series of thirty strains of Staphylococcus aureus and albus there was correspondence between penicillinase production, 'cell sensitivity' to penicillin and penicillin lysis, but no relation between these characteristics and the results of eleven routine tests for pigmentation, $\beta$-haemolysis, coagulase formation and various biochemical reactions.

It has been recognized for some time that staphylococci may be divided into those which do and those which do not produce a penicillinase (Hobby \& Dawson, 1944; Spink, Ferris \& Vivino, 1944; Kirby, 1945a,b). Also, staphylococci are often tested for their penicillin sensitivity (tolerance) and classified as 'sensitive' or 'insensitive'. The literature contains a number of references to another staphylococcal characteristic vis-à-vis penicillin which might be used as a third criterion of classification, namely penicillin lysis.

The phenomena of autolysis and lysis by drugs are classical in bacteriology; the subject has recently been reviewed by Pulvertaft \& Lumb (1948). Penicillin lysis of staphylococci was observed by Fleming (1929), Smith \& Hay (1942) and Gardner (1945), and related to turbidity measurements by Chain, Duthie \& Callow (1945) and Kirby (1945a). Knox (1945) and Todd (1945) related the occurrence of lysis to the growth-phase of the organisms, as did Chain et al. by making cell-counts and viable counts of their cultures. A comparison between the chemical activity of areas of lysis and areas of growth was made on assay plates by Pratt \& Dufrenoy (1947), who showed that lipids were liberated in areas of lysis, and hydrolysed into fatty acids. The resistance of certain staphylococcal strains to lysis was noted by Kirby and by Knox, being associated with relative tolerance of the bactericidal action of penicillin. Kirby (1945b) also demonstrated the presence of penicillinase in unlysable, resistant strains. In view of the reports of Kirby and of Knox, a study was made of the correspondence between penicillin lysis, penicillinase production and penicillin 'cell sensitivity' (Luria, 1946) of a small number of strains of staphylococci, which were also tested for other characteristics.

\section{METHODS}

Thirty-seven strains of staphylococci were investigated. They were mostly obtained from routine clinical material, and were stored at room temperature on nutrient agar slopes, and tested in batches. Seven strains are reported separately, as they produced so much penicillinase that no lysis was observable with the penicillin concentration employed in the test. The main investigation is therefore concerned with thirty strains. 


\section{Technique of plate inoculations}

Pyrex Petri dishes, $15 \mathrm{~cm}$. diameter, were used. Particular attention was paid to standardizing the inocula on all the plate cultures; for seeding, the following modification was evolved of Heatley's (1947) Pasteur pipette pen. Standard thin-wall glass capillary tubing of $1.5 \mathrm{~mm}$. external diameter was obtained in $4 \mathrm{in}$. lengths, the terminal centimetre of each bent to make an angle of about $100^{\circ}$, and the cut edges of the tip of the bent portion smoothed without decrease in lumen. A pivoted holder was devised for seeding from the capillaries under standard conditions which deposited a repeatable, even inoculum (Fig. 1).

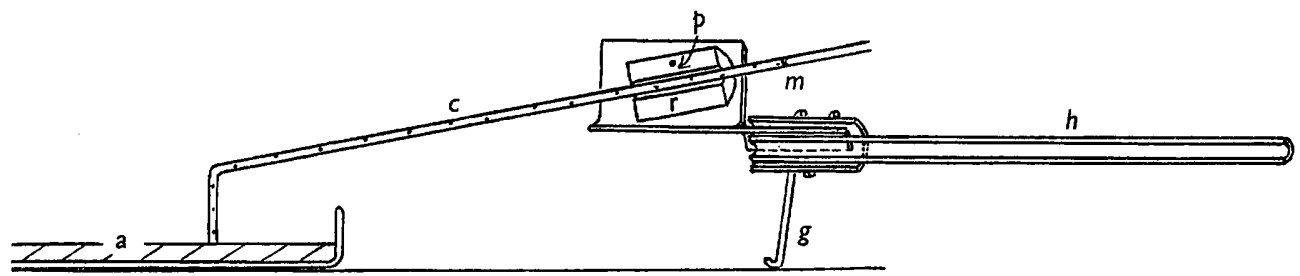

Fig. 1. Capillary pipette pen. The bent capillary $c$ is gripped by the section of thick-walled rubber tubing $r$ which is pivoted at $p$. The capillary is filled to the mark $m$, and the tip rests vertically under its own weight on the surface of the agar $a$. By means of the holder $h$ the tip of the capillary is drawn over the agar at right-angles to the plane of the diagram, with the guides $g$ (only one of the pair is shown) resting on the bench. Provided the height $a p$ is constant, the angle of $h$ is immaterial.

The estimations of cell sensitivity were made with light and heavy streaks. The light streaks were 1/100 to 1/1000 dilutions of 24 hr. broth cultures, and the heavy streaks the centrifuged deposit from similar cultures suspended in a few drops of broth (Ingram, 1951).

\section{Bacteriological tests}

The following investigations were made on all strains:

Plate cultures (heavy streaks). $\beta$-Haemolysis and pigment production on human blood agar $(0.5 \% \mathrm{NaCl})$; coagulase and pigment production on $20 \%$ human plasma agar (Reid \& Jackson, 1945). These plates were incubated at $37^{\circ}$ and read after c. $24 \mathrm{hr}$.

Liquid cultures. Coagulase production by a $6 \mathrm{hr}$. tube method used in this laboratory as a routine; production of acid and clot in Hiss's serum water containing glucose, sucrose, lactose or mannitol; and production of acid and curd in litmus milk. These were incubated at $37^{\circ}$ and read daily for a week. Strains were classified as coagulase positive when either the plate or the tube test was positive. Similarly, classification was by the darker pigment produced on either blood agar or plasma agar.

Stab culture. Liquefaction of gelatin at room temperature, read daily for a week. 


\section{Penicillin tests}

The following investigations were made:

Penicillin-dilution plates. Following the techniques of Foster \& Woodruff (1943) and Frisk (1945), a series of penicillin dilutions were made in molten agar, and poured in Petri dishes. When set, surface light streaks were made with the modified Heatley pen. After incubation the results were recorded by noting the highest concentration of penicillin that permitted growth, and the lowest that inhibited growth, in the series of plates that was used, for example, '0.01-0.02'. This value was taken as the cell sensitivity, but was not determined for all the strains because the technique was unduly time-consuming.

Penicillin-diffusion plates. Suggested by the technique of Mohs (1945), and following Fleming's (1929) and Barber's (1947a) ditch-plate principle, heavy and light streaks of each strain were sown with the pen in parallel lines on the surface of plain agar plates, each also carrying three streaks of the Oxford staphylococcus 'H' (N.C.T.C. 6571). Across each plate at right-angles to the streaks was then laid a strip of thick blotting-paper evenly saturated with penicillin solution of 10,000 units $/ \mathrm{ml}$. After incubation the results were read as follows: measurement was made of the distance from the edges of the penicillin reservoir to the limits of growth in each streak, and the distances obtained with the test organisms were expressed as a percentage of the mean distance in the three control streaks on the same plate. This value is referred to as the percentage inhibited distance. The measure does not imply that a linear relationship exists between penicillin concentration and 'inhibited distance' (this relation is usually taken to be logarithmic), but the convention is simple and sufficient to establish large differences between groups. The percentage inhibited distances of the light streaks were regarded as an approximate indication of the 'cell sensitivity' of those strains in which this value was not determined by the plate-dilution method.

The heavy streaks on the same preparations were used for the observation of lysis, which when present was found at the junction of free and inhibited growth (cf. Fleming, 1929; Knox, 1945).

\section{Method of soaking linear penicillin reservoirs}

Thick blotting-paper is cut into suitable strips of even width which are saturated by immersion in penicillin solution, and the excess run off. They are drained by laying on a firm pad of absorbent material and gently pressed into contact. When it is seen that excess of moisture has been taken up, usually in 2 or 3 sec., the strips are transferred to the assay plate. An ordinary asbestos cooking mat has been found satisfactory for the absorbent pad, and it can be sterilized in the flame from time to time. The method was shown to produce an even saturation of individual strips and a reproducible impregnation from strip to strip by statistical analysis of experiments with multiple streaks. Fig. 2 shows the clear zones produced on surface pre-seeded plates by strips soaked in various penicillin solutions, and Table 1 gives the analysis of variance for the test of strip to strip reproducibility. 
Table 1. Analysis of variance for test of strip to strip variability

\begin{tabular}{|c|c|c|c|c|}
\hline Contribution & $\begin{array}{l}\text { Degrees } \\
\text { of } \\
\text { freedom }\end{array}$ & $\begin{array}{c}\text { Sum } \\
\text { of } \\
\text { squares }\end{array}$ & $\begin{array}{c}\text { Mean } \\
\text { square } \\
\text { (variance) }\end{array}$ & $\begin{array}{l}\text { Variance } \\
\text { ratio }\end{array}$ \\
\hline doses: Regression & 1 & $476 \cdot 58$ & $476 \cdot 58$ & 1604 \\
\hline About regression & 6 & $3 \cdot 81$ & $0 \cdot 635$ & $2 \cdot 14$ \\
\hline ses totals & 7 & $480 \cdot 39$ & & \\
\hline doses & 40 & $11 \cdot 89$ & 0.297 & 1.00 \\
\hline
\end{tabular}

The $P=0 \cdot 05$ limit for $n_{1}=6, n_{2}=40$ is $2 \cdot 34$, so deviations from regression are not significant. The variance ration for linear regression is clearly very significant.
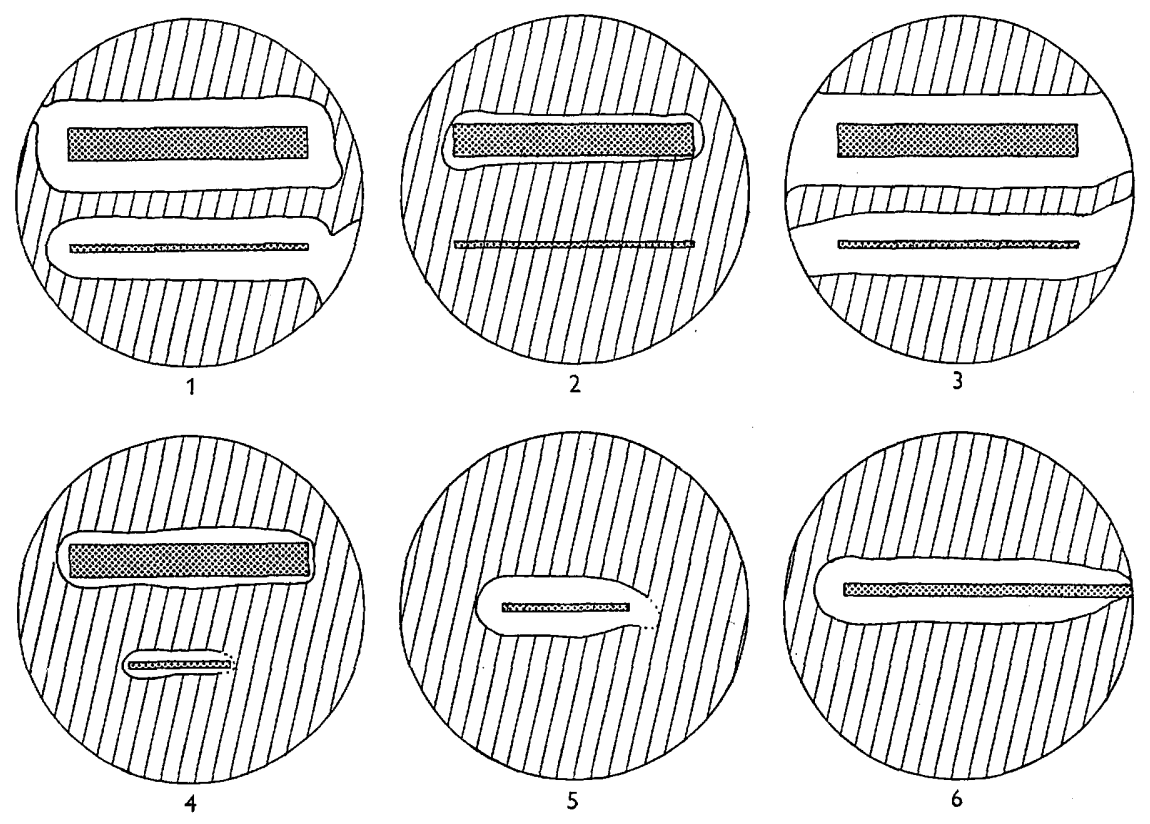

DID $\begin{aligned} & \text { Free bacterial } \\ & \text { growth }\end{aligned}$
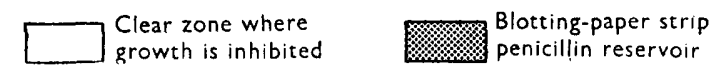

Fig. 2. Tracings of the outlines of growth after incubating evenly-seeded agar plates (Staph. aureus) carrying blotting-paper strips soaked in penicillin solutions. The broken outlines indicate an indistinct margin between areas of free and inhibited growth.

Plate 1. Two strips soaked and drained by the described technique, carrying 10,000 units $/ \mathrm{ml}$. Wide clear zones were formed, regular over a long central portion, and of the same width with both strips. Irregularities were found at the ends of the strips, with the solution 'creeping' to the edge of the plate.

Plate 2. Two strips similarly soaked, carrying $100 \mathrm{units} / \mathrm{ml}$. An uneven, narrow zone was formed round the wide strip, and no zone round the narrow strip.

Plates 3 and 4. Two strips each similar in size and penicillin concentration to those on plates 1 and 2 , soaked but not drained on the absorbent pad: excess solution was merely run off before the strips were laid on the plates. Plate 3 gave similar appearances to plate 1, except that there was more 'creeping'. Plate 4 showed a substantially similar result to plate 2 , the difference in zone width being presumably due to the greater absolute quantity of penicillin carried over in the reservoirs.

Plates 5 and 6. One end only of each strip soaked in penicillin solution 10,000 units/ ml., and not drained. 'Creeping' had occurred in both strips, so that some clear zone was seen along the whole length of the strips. Gross irregularities were obvious. 


\section{Test of strip-to-strip reproducibility: analysis of variance}

Eight strips were soaked by the described technique in a twofold dilution series of penicillin solutions containing 10,000,5000, 2500, .., 78 units $/ \mathrm{ml}$. and laid at right angles over parallel streaks of a penicillin-sensitive Staphylococcus aureus (N.C.T.C. 6571) on a $27 \times 27 \mathrm{~cm}$. agar slab, so that each strip crossed six streaks. The paired responses obtained from each crossing were averaged. The above analysis (Table $\mathrm{I}$ ) of the forty-eight means shows that the impregnation was sufficiently well reproduced from strip to strip for the deviations of strip means from the general regression to be less than the scatter of the readings within individual strips. The correlation coefficient, $r$, was $+0 \cdot 9960$. The actual deviations of the strip means from the regression line were: $+\mathbf{0} \cdot 08,-\mathbf{0} \cdot 12$, $+0 \cdot 06,-0 \cdot 36,+0 \cdot 25,+0 \cdot 53,-0 \cdot 10$ and $-0 \cdot 36 \mathrm{~mm}$. The standard deviations of individual strip means lay in the range $0.831-0.386 \mathrm{~cm}$., mean $0.578 \mathrm{~cm}$. (coefficients of variation $3 \cdot 34-1 \cdot 42 \%$, mean $2 \cdot 47 \%$ ).

Penicillinase tests. Twenty-six strains were tested qualitatively for the production of penicillinase, either by Kirby's $(1945 b)$ adaptation of Harper's (1943) extraction, or by a 'hole-plate' or 'pad-plate' technique similar in principle to that used by Barber (1947a), or by a 'parallel-streak' method similar to that of W. Hayes (unpublished).

\section{RESULTS}

Twenty-nine strains were classified without difficulty by the criterion of lysis; a thirtieth strain could not be classified. Fig. 3 shows the characteristic appearances of the two types of strain when growing at right-angles to a linear penicillin source. Nineteen strains were lysed and ten were not.

Bacteriological tests. The differences between strains in the bacteriological tests were entirely uncorrelated with lysis; in particular, yellow and white pigmented strains and coagulase positive and negative strains occurred in both groups.

Special penicillin tests. By contrast, the penicillin tests showed a consistent distinction between the two groups. The nineteen lysed strains were all relatively sensitive; the percentage inhibited distances of the light inocula lay within the range $88-112 \%$, that is to say, these organisms all had about the 'sensitivity' of the Oxford strain. In eight strains of this group the cell sensitivity was accurately determined by the plate-dilution test, and lay within the range $0.01-0.02$ to $0.05-0.06$ (the value for the Oxford strain was $0.01-0.02$ ). Similarly, eleven strains of this group were directly tested for penicillinase production and all gave a negative result; the percentage inhibited distances of the heavy streaks lay within the range $59-68 \%$.

The ten unlysed strains, on the other hand, were all relatively resistant to penicillin; of nine tested by light streaks against the linear penicillin source, the range of percentage inhibited distances lay between 52 and $63 \%$. Eight strains (including the one not tested by streak) of this group whose cell sensitivities were accurately determined by the plate-dilution test, gave values of $0 \cdot 2-0 \cdot 4$ to $0 \cdot 6-0 \cdot 8 \mathrm{unit} / \mathrm{ml}$. Penicillinase production was tested directly in 
eight strains; all were positive. The percentage inhibited distances with the heavy inocula varied in nine strains from 7 to $53 \%$.

Additional observations on very resistant strains. Seven further strains were fully tested which differed from the above strains in the lysis test, since heavy streaks grew right up to the penicillin source, so that the junction of free and inhibited growth could not be observed. All these seven strains produced

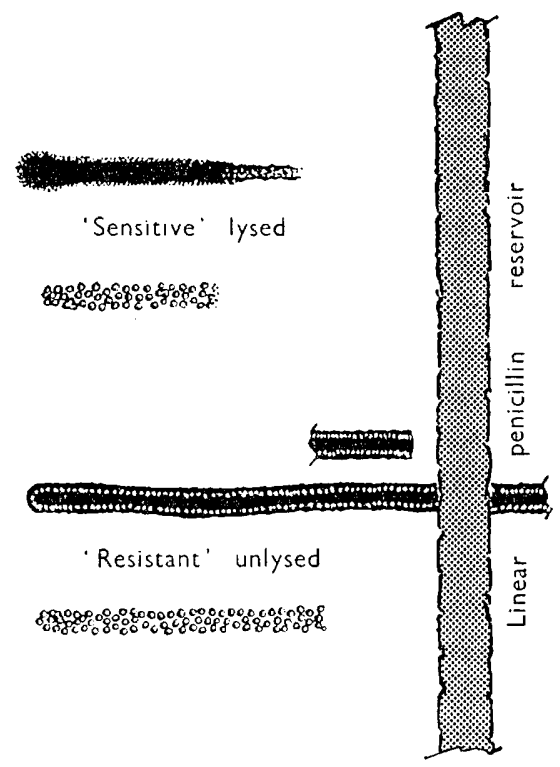

Fig. 3. (Scale $\frac{3}{2}$ ). Heavy and light streaks of sensitive and resistant strains of Staph. aureus evenly sown at right-angles to a linear penicillin reservoir. Lysis is shown by the sensitive strain (which does not produce penicillinase) in the 'comet-tail' lying between normal growth and the inhibited portion of the streak. It is best seen in the heavy streak. The resistant, penicillinase-producing strain may grow right up to a reservoir of low concentration, but when inhibited at a distance from a higher concentration (as in the short heavy, streak immediately above the 'resistant' unlysed streaks) shows no lysis.

penicillinase, and it is thought that they produced sufficient enzyme to enable them to grow freely in the highest concentration of the drug which they encountered on the plate. Four strains were tested by the plate-dilution method; the cell sensitivities varied from $0 \cdot 6-2 \cdot 0$ to $1 \cdot 0-3 \cdot 0$ units $/ \mathrm{ml}$. In six instances the percentage inhibited distance was determined with light streaks, and lay in the range $40-63 \%$ (five within the range $46-63 \%$ ).

\section{DISCUSSION}

In this small sample it seems that there is a division of staphylococci into two groups: (a) those whose cells are sensitive to penicillin, do not produce a penicillin-destroying enzyme, and are lysed by penicillin; $(b)$ those whose cells are resistant to penicillin, are not lysed by it and produce a penicillinase. The second group of course includes only those staphylococci naturally tolerant of penicillin, and not those trained to tolerance by the artificial selection of 
variants, which are usually of low virulence and are uncommon in clinical lesions.

The failure to detect any differences in the biochemical and cultural characteristics of the two groups agrees with Barber's (1947b) report that such tests fail to distinguish penicillinase-producing strains from those which do not.

There is a large number of tests now in use for the routine clinical determination of penicillin sensitivity (tolerance). Many of these depend on observing the growth of cultures in relation to a penicillin source on an agar plate, and applying an arbitrary distinction between those considered sensitive and those classed as resistant. It is, of course, realized that these tests do not usually allow for the possible effect of penicillinase upon the result of the test, and so in using such techniques one is in doubt of what is really being assessed.

Spink et al. (1944), Luria (1946) and Barber (1947 b) showed that the experimental value obtained for the penicillin tolerance of a penicillinase-producing staphylococcus depended on the size of the inoculum, whereas with a nonproducer of penicillinase the inoculum size did not affect the tolerance value. In any measurement depending on the diffusion of penicillin through agar, the depth of the agar (Hayes, 1945), the composition of the batch of medium (Pulvertaft, Greening \& Haynes, 1947), the volume of solution in the penicillin reservoir (Grenfell, Legge \& White, 1947) and perhaps other factors will affect the value obtained, irrespective of the concentration of penicillin against which the organism is tested. In the present work precautions were taken to standardize the inoculum (light streaks) for the penicillin tolerance tests, and to use so small an inoculum that the effect of penicillinase would be inappreciable.

The tenfold difference observed between the cell-sensitivity ranges of the two smaller groups of strains tested by dilution plates is of the same order as that found by Bondi \& Dietz (1945), who examined penicillinase production and penicillin tolerance (also by penicillin dilution plates) in 115 strains of staphylococci; although fairly heavy inocula were used, it is probably significant that their strains also fell into two groups whose tolerance ranges did not overlap. All the more tolerant strains (16) and none of the less tolerant (99) produced the enzyme.

Evidence on the production of penicillinase was also thought to be provided by the percentage inhibited distances of the heavy streaks. This value was much less in the strains known to produce the enzyme than in those not producing it, and the greater diminution was thought to be due to it. This agrees with the findings of Spink et al., of Luria and of Barber quoted above. The strains which did not produce enzyme also showed some diminution of the percentage inhibited distance in heavy streak; this is apparently at variance with the findings of those authors. It is therefore possible that these very dense streaks contained enough more tolerant cells to have produced a visible growth nearer to the penicillin source than was possible in the corresponding light streaks. Numerical data on this point will be presented elsewhere (Ingram, Foxell \& Armitage, unpublished). The above finding is in accord with that of Gilson \& Pairker (1948), who reported an increase in the tolerance range of staphylococci not producing penicillinase from $0.008-0 \cdot 12 \mathrm{unit} / \mathrm{ml}$. to 
0.016-1.0 unit $/ \mathrm{ml}$. when the inoculum was increased by only a hundred-fold. By comparison, the same inoculum increase with the penicillinase-producers gave an increase in the tolerance range from $0 \cdot 06-4 \cdot 0 \mathrm{units} / \mathrm{ml}$. to $0 \cdot 06-500$ units $/ \mathrm{ml}$.

If the results of the present investigation are confirmed, either penicillin lysis or penicillinase production (as suggested by Barber, 1948) would be satisfactory as single criteria of the penicillin sensitivity of staphylococci for ordinary clinical purposes. These characteristics are not quantitatively determined by the arbitrary conditions of a given plate, as is, say, the 'inhibited distance' from the penicillin source. Barber (1948) has pointed out that the presence or absence of the ability to destroy penicillin is of much greater clinical significance than the exact sensitivity of the strain.

A high concentration of penicillin should be present in the diffusion source if observations are to be made on the junction of normal and inhibited growth in the case of powerful penicillinase producers.

Grateful acknowledgement is made to Prof. W. G. Barnard and the Council of St Thomas's Hospital Medical School for a Bursary during the tenure of which this investigation was carried out; to Dr J. Bamforth for laboratory facilities; to Dr V.D. Allison for phage typing and Dr A. W. H. Foxell for penicillinase tests ; to P. Armitage, Esq., of the Medical Research Council Statistical Research Unit, for kindly performing the computations for the strip-to-strip reproducibility test of the strip-soaking investigation; to Dr N. G. Heatley, Dr Mary Barber and Dr R. L. Vollum for helpful criticism of MS.; to Dr W. A. R. Thomson of Boots Pure Drug Co. for the gift of penicillin; to James A. Jobling Co. for the gift of glass capillaries; to the late $\mathrm{Mr}$ Radford, and to Miss Whitley and Miss King for technical assistance, and to the Hospital carpenters and engineers, especially Mr Brignall, who constructed the base and cover of the apparatus used for the $27 \times 27 \mathrm{~cm}$. agar slab.

\section{REFERENCES}

Barber, M. (1947a). Staphylococcal infection due to penicillin-resistant strains. Brit. med. J. ii, 863.

Barber, M. (1947b). Coagulase-positive staphylococci resistant to penicillin. J. Path. Bact. 59, 373.

Barber, M. (1948). The present position of penicillin. St Thom. Hosp. Gaz. 46, 162.

Bondi, A. \& Dietz, C. C. (1945). Penicillin resistant staphylococci. Proc. Soc. exp. Biol., N.Y. 60, 55.

Chain, E., Duthre, E. S. \& Callow, D. (1945). Bactericidal and bacteriolytic action of penicillin on the staphylococcus. Lancet, $\mathrm{i}, 652$.

Fleming, A. (1929). On the antibacterial action of cultures of a penicillium, with special reference to their use in the isolation of B. Influenzae. Brit. J. exp. Path. $10,226$.

Foster, J. W. \& Woodruff, H. B. (1943). Microbiological aspects of penicillin: 1. Methods of assay. J. Bact. 46, 187.

Frisk, A. R. (1945). Bestamning av penicillinkoncentration och penicillin-kanskighet. Nord. med. (Hygiea). 28, 2249.

Gardner, A. D. (1945). Microscopical effect of penicillin on spores and vegetative cells of bacilli. Lancet, i, 658.

Gilson, B. St C. \& Parker, R. F. (1948). Staphylococcal penicillinase: characteristics of the enzyme and its distribution. J. Bact. 55, 801. 
Grenfell, E., Legge, B. J. \& White, T. (1947). Microbiological aspects of the submerged-culture production of penicillin on a pilot-plant scale. $J$. gen. Microbiol. 1, 171.

HARPER, G. J. (1943). Inhibition of penicillin in routine culture media. Lancet, ii, 569.

HAYES, W. (1945). The effect of agar depth in the plate method for the assay of penicillin. J. Path. Bact. 57, 457.

Heatley, N. G. (1947). A simple plate method for multiple tests of the antibacterial activity of many bacteria against other bacterial strains. J. gen. Microbiol. 1, 168.

Hobвy, G. L. \& Dawson, M. H. (1944). Bacteriostatic action of penicillin on haemolytic streptococci in vitro. Proc. Soc. exp. Biol., N.Y., 56, 178.

INGRAM, G. I. C. (1951). Formation of clear zones with sensitive and resistant Staphylococcus aureus in penicillin plate assays. J. gen. Microbiol. 5, 22.

KirBy, W. M. M. (1945a). Bacteriostatic and lytic actions of penicillin on sensitive and resistant staphylococci. J. clin. Invest. 24, 165.

Krrby, W. M. M. (1945b). Properties of a penicillin inactivator extracted from penicillin-resistant staphylococci. J. clin. Invest. 24, 170.

KNox, R. (1945). Effect of penicillin on cultures in liquid and solid media. Lancet, i, 559.

LURIA, S. E. (1946). A test for penicillin sensitivity and resistance in staphylococcus. Proc. Soc. exp. Biol., N.Y., 61, 46.

Mонs, F. E. (1945). A simple quantitative test for the penicillin sensitivity of bacteria; the 'radial streak' method. J. Lab. clin. Med. 30, 800.

Pratt, R. \& Dufrenoy, J. (1947). Cytochemical mechanisms of penicillin action. II. Changes in reactions of Staphylococcus aureus to vital dyes. J. Bact. 54, 127.

Pulvertaft, R. J. V., Greening, J. R. \& Haynes, J. A. (1947). Liesegang rings and antiseptics. J. Path. Bact. 59, 293.

Pulvertaft, R. J. V. \& Lumb, G. D. (1948). Bacterial lysis and antiseptics. $J$. Hyg., Camb., 46, 62.

Reid, J. D. \& JAckson, R. M. (1945). An improved method for determining the coagulase activity of staphylococci by means of the plasma agar plate. J. Lab. clin. Med. 30, 155.

Smrth, L. D. \& HAY, T. (1942). The effect of penicillin on the growth and morphology of Staphylococcus aureus. J. Franklin Inst. 233, 598.

Spink, W. W., Ferris, V. \& Vivino, J. J. (1944). Comparative in vitro resistance of staphylococci to penicillin and to sodium sulphathiazole. Proc. Soc. exp. Biol., N.Y., 55, 207.

Todd, E. W. (1945). Bacteriolytic action of penicillin. Lancet, i, 74.

(Received 15 February 1950) 